\title{
A Diet Quality Index for Pregnancy detects variation in diet and differences by sociodemographic factors
}

\author{
Lisa M Bodnar ${ }^{1,2, *}$ and Anna Maria Siega-Riz ${ }^{1,2,3}$ \\ 'Department of Nutrition, University of North Carolina, Schools of Public Health and Medicine, Chapel Hill, NC, \\ USA: ${ }^{2}$ Carolina Population Center, Chapel Hill, NC, USA: ${ }^{3}$ Department of Maternal and Child Health, University of \\ North Carolina, School of Public Health, Chapel Hill, NC, USA
}

Submitted 17 October 2001: Accepted 28 March 2002

\begin{abstract}
Objective: Methods currently used to assess nutritional status during pregnancy have limitations if one wishes to examine the overall quality of the diet. A Diet Quality Index for Pregnancy (DQI-P) was developed to reflect current nutritional recommendations for pregnancy and national dietary guidelines.

Design: Dietary intake was assessed during the second trimester using a foodfrequency questionnaire. The DQI-P includes eight components: \% recommended servings of grains, vegetables and fruits, \% recommendations for folate, iron and calcium, \% energy from fat, and meal/snack patterning score. Scores can range from 0 to 80; each component contributed 10 points.

Setting: Two public prenatal clinics in central North Carolina.

Subjects: $N=2063$ pregnant women who participated in the Pregnancy, Infection, and Nutrition (PIN) Study.

Results: The DQI-P quantitatively differentiated diets. The mean score for the population was 56.0 (standard deviation 12.0). Women who were $>30$ years old, $>350 \%$ of poverty, nulliparous and high school graduates had significantly higher overall DQI-P scores. Higher percentages of recommended vegetable servings were consumed by higher-income, older and better-educated women. Greater percentages of recommended intakes of folate and iron were seen among black, low-income and nulliparous women. Higher iron intakes were also seen among women who graduated high school and were less than 30 years old. Other differences were observed for intake of fat and meal/snack pattern. Because this index was based on national recommendations, the DQI-P may be a useful tool for research and public health settings to evaluating overall diet quality of pregnant women.
\end{abstract}

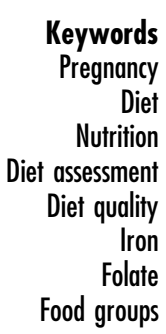

Nutritional status during pregnancy is an important determinant of birth outcomes ${ }^{1,2}$. Methods currently used to assess nutritional status during pregnancy have limitations if one wishes to examine the overall quality of the diet. Valid biochemical indices for assessing specific nutrients, such as the use of haemoglobin concentration as a marker of iron status, are available for a limited number of nutrients and have numerous shortcomings, including the inability to assess more than one nutrient at a time. Gestational weight gain assessments can determine if caloric intake is adequate, but cannot estimate adequacy of intake of specific nutrients or food groups. Dietary assessment tools such as food records, multiple 24-hour dietary recalls or food-frequency questionnaires can be used to assess diet quality; however, because these tools are multidimensional, a meaningful interpretation of diet quality is not possible unless results are simplified into a composite score.
Measures of overall diet quality have been developed for adults ${ }^{3-6}$ based on the Dietary Guidelines for Americans $^{7}$ and the Food Guide Pyramid ${ }^{8}$. The two most commonly used indices, the Diet Quality Index ${ }^{3,4}$ and the Healthy Eating Index ${ }^{5,6}$, have the advantage of explaining the full complexity of the diet by including both food and nutrient components, yet are not highly correlated with total energy intake. Furthermore, these measures reflect variation in both the components upon which the indices are based as well as other dietary characteristics not directly measured.

Despite the many advantages of the indices created previously, they cannot apply to the pregnant state because dietary recommendations differ. Therefore, a distinct tool that incorporates all diet quality measures for pregnancy must be created. The objective for this project was to develop a tool that measures dietary quality and reflects the most current nutritional recommendations for 
pregnancy. Additionally, we assessed differences in diet quality by sociodemographic characteristics.

\section{Population and methods}

\section{Population and data collection methods}

This study used data from the Pregnancy, Infection, and Nutrition (PIN) Study, a prospective cohort study of the determinants of pre-term birth. The study recruits predominantly lower- to middle-income women from four prenatal care clinics in two settings: the University of North Carolina Resident and Private Physician Obstetrics Clinic and the Wake County Department of Human Services and Wake Area Health Education Center Prenatal Care Clinics. Women were recruited into the study from 24 to 29 weeks of gestation. Several questionnaires were selfadministered at that time including a food-frequency questionnaire for dietary intake. Subjects were also interviewed by telephone to obtain sociodemographic data, health habits and previous as well as current medical history. Birth outcome information was obtained from hospital delivery logs. The procedures followed for this study were in accordance with the ethical standards of the Institutional Review Board of the University of North Carolina School of Medicine and Wake Medical Center.

\section{Ascertaining dietary data and its analysis}

In the PIN Study dietary intake was assessed at 26 to 28 weeks of gestation using a 120-item modified NCI-Block Food Frequency Questionnaire (FFQ). This FFQ had updated nutrient values based on data from the US Department of Agriculture's (USDA) 1994-96 Continuing Survey of Food Intake by Individuals for women aged 1944 years ${ }^{9}$ and updated folate values for fortified foods from the USDA's 1998 nutrient database ${ }^{10}$. The PIN FFQ captures usual dietary intake in the three-month time period of the second trimester of pregnancy. This tool has been validated in a variety of populations ${ }^{11-14}$, including our own. Energy-adjusted deattenuated Pearson correlation coefficients for the nutrients in this index are as follows: iron, $r=0.53$; folate, $r=0.45$; calcium, $r=0.51$; fat, $r=0.43$. Food groups have not been validated as such. The FFQ is designed to be self-administered, requiring about 20 to 30 minutes for completion. Slight modification has been made to include local foods, to focus on a three-month time period, and to incorporate the latest recommendations for improving the quality of information collected ${ }^{15}$. Because this FFQ is semiquantitative, it provides a projection of the quantity of nutrients consumed. To ascertain information on meal and snack patterning, the FFQ included two questions asking subjects to report usual number of meals (breakfast, lunch, dinner) and snacks consumed per day, as well as the timing of those eating occasions. Results are presented for 2063 women who provided complete dietary data and some information on sociodemographic characteristics and health behaviours.

DietSys 4.0 software was used to analyse the questionnaires ${ }^{16}$. The software provides estimates of usual intake of 33 nutrients and calculates daily frequency and daily gram amounts for each individual food item and identifies up to 20 food groups that can be modified by the investigator. In addition to modifying several food groups that already existed, we created new food groups in order to calculate servings of food groups that corresponded to the Food Guide Pyramid ${ }^{8}$. When modifying food groups, we did not include french fries or fried potatoes as vegetables, as recommended by the National Cancer Institute ${ }^{17}$.

The DietSys program calculates the total grams of food consumed for each food group and provides the gram weight for each serving size (small, medium, large) of the approximately 120 food items. For the three food groups used in the index (grains, vegetables and fruits), we calculated an average serving size using the gram weight of the medium serving size as specified in the FFQ. To calculate the number of servings each individual consumed, we divided the total grams of food consumed for the food group by the average serving size. To prevent overestimating portion sizes of fruit by including the weight of fruit juices, we conducted this analysis separately for fruit juices and solid fruits. The average number of servings of juice was then added to the number of fruits to obtain total servings for the fruit food group. FFQ line items representing mixed dishes, such as 'mixed dishes with cheese' or 'mixed dishes with chicken', could contribute to the grain food group; but, since we had no way of assessing the inclusion of vegetables and fruits in these dishes, they did not contribute to these food groups.

\section{Structure and development process for the DQI-P}

The DQI-P was based on eight dietary characteristics as shown in Table 1 . The first three components reflect the dietary adequacy of grain, vegetable and fruit intakes based upon the Dietary Guidelines for Americans ${ }^{7}$ and the Food Guide Pyramid ${ }^{8}$. Because energy intake determines the number of recommended servings for all three food groups, we applied food group serving recommendations from the Pyramid to five categories of energy intake: $\leq 6694 \mathrm{~kJ} \quad(\leq 1600 \mathrm{kcal}), \quad 6695-<7949 \mathrm{~kJ} \quad(1601-$ $<1900 \mathrm{kcal}), \quad 7949-<10460 \mathrm{~kJ} \quad(1900-<2500 \mathrm{kcal})$, $10460-<11715 \mathrm{~kJ}(2500-<2800 \mathrm{kcal})$ and $\geq 11715 \mathrm{~kJ}$ $(\geq 2800 \mathrm{kcal})$. This was then used to calculate the percentage of recommended servings consumed. Despite the recommendation to consume $10460 \mathrm{~kJ}$ (2500 kcal) during pregnancy ${ }^{18}$, we chose not to base recommended servings on this energy level. Literature has shown that actual energy intakes in pregnancy vary considerably because metabolic response to pregnancy differs substantially among women ${ }^{19}$.

The next three components of the DQI-P reflect the 
Table 1 Dietary components included in the Diet Quality Index for Pregnancy, $n=2063$

\begin{tabular}{|c|c|c|c|}
\hline Component & Score & Score categories & $\begin{array}{l}\% \text { Population } \\
\text { in subgroup }\end{array}$ \\
\hline \multirow[t]{3}{*}{$6-11$ servings of grains per day, $\%$ recommended servings ${ }^{1,2}$} & \multirow[t]{3}{*}{$0-10$} & $\geq 100 \%$ & 1.2 \\
\hline & & $99 \%-50 \%$ & 19.5 \\
\hline & & $<50 \%$ & 79.3 \\
\hline \multirow{3}{*}{$3-5$ servings of vegetables per day, $\%$ recommended servings ${ }^{1,2}$} & \multirow[t]{3}{*}{$0-10$} & $\geq 100 \%$ & 37.4 \\
\hline & & $99 \%-50 \%$ & 36.7 \\
\hline & & $<50 \%$ & 25.9 \\
\hline \multirow{3}{*}{$2-4$ servings of fruits per day, $\%$ recommended servings ${ }^{1,2}$} & \multirow[t]{3}{*}{$0-10$} & $\geq 100 \%$ & 51.3 \\
\hline & & $99 \%-50 \%$ & 25.8 \\
\hline & & $<50 \%$ & 23.0 \\
\hline \multirow[t]{3}{*}{ Folate intake as $\% \mathrm{RDA}^{2,3}$} & \multirow[t]{3}{*}{$0-10$} & $\geq 100 \%$ & 43.8 \\
\hline & & $99 \%-50 \%$ & 43.8 \\
\hline & & $<50 \%$ & 12.4 \\
\hline \multirow[t]{3}{*}{ Iron intake as \% RDA ${ }^{2}$} & \multirow[t]{3}{*}{$0-10$} & $\geq 100 \%$ & 19.4 \\
\hline & & $99 \%-50 \%$ & 52.2 \\
\hline & & $<50 \%$ & 28.4 \\
\hline \multirow[t]{3}{*}{ Calcium intake as $\%$ Al for age ${ }^{2}$} & \multirow[t]{3}{*}{$0-10$} & $\geq 100 \%$ & 56.5 \\
\hline & & $99 \%-50 \%$ & 33.5 \\
\hline & & $<50 \%$ & 10.0 \\
\hline \multirow[t]{4}{*}{ Total fat $\leq 30 \%$ energy intake ${ }^{4}$} & \multirow[t]{4}{*}{$0-10$} & $\leq 30 \%$ & 28.8 \\
\hline & & $>30 \%, \leq 35 \%$ & 30.9 \\
\hline & & $>35 \%, \leq 40 \%$ & 25.3 \\
\hline & & $>40 \%$ & 15.1 \\
\hline \multirow[t]{3}{*}{ Meal pattern ${ }^{5}$} & \multirow[t]{3}{*}{$0-10$} & 3 meals/2 snacks & 71.6 \\
\hline & & 3 meals/0-1 snack(s) or 2 meals/2 snacks & 12.4 \\
\hline & & 2 meals/0-1 snack(s) or 1 meal/snacks & 16.1 \\
\hline
\end{tabular}

RDA - Recommended Dietary Allowance; Al - Adequate Intake.

${ }_{1}^{1}$ Based on Food Guide Pyramid ${ }^{8}$ recommendations for diets containing $\leq 1600,1601-<1900,1900-<2500,2500-<2800$ and $\geq 2800 \mathrm{kcal}$.

${ }^{2}$ Used as a continuous percentage ( $0 \%$ to $\left.100 \%\right)$ corresponding to a continuous DQI-P score of 0 to 10 points.

${ }^{3}$ As dietary folate equivalents.

${ }^{4}$ Scoring based on the following categories: $\leq 30 \%=10$ points; $>30 \%, \leq 35 \%=7$ points, $>35 \%, \leq 40 \%=4$ points, $>40 \%=0$ points.

${ }^{5}$ Scoring based on the following categories: 3 meals $/ 2$ snacks $=10$ points; 3 meals/0-1 snack(s) or 2 meals $/ 2$ snacks $=5$ points; 2 meals $/ 0-1$ snack(s) or $1 \mathrm{meal} / \mathrm{snacks}=0$ points.

intake of nutrients particularly important for pregnancy: folate, iron and calcium. These nutrients represent dietary intake exclusive of vitamin/mineral supplements. Because the DQI-P is used to assess the adequacy of individuals' diets, we compared intakes to the Recommended Dietary Allowance (RDA), the value at or above which there is low probability of dietary inadequacy ${ }^{20}$. The RDA for folate is $600 \mu \mathrm{g} \mathrm{day}^{-1}$ of dietary folate equivalents (DFEs) ${ }^{21}$. DFEs take into account the increased bioavailability of synthetic folic acid compared with naturally occurring food folate. We estimated each woman's DFE intake by first calculating the percentage of total grams of food contributing to folate intake coming from fortified food sources. This proportion of intake was then multiplied by the total micrograms of dietary folate in order to estimate the amount of synthetic folic acid consumed. We then multiplied the resulting micrograms of folic acid by 1.7 , as recommended by Yates et $a l^{22}$, to account for the increased bioavailability. Finally, this value was added to the remaining micrograms of folate from natural sources to estimate the DFEs.

Adequacy of iron intake is based on the RDA of $27 \mathrm{mgday}^{-1}$ for pregnancy ${ }^{23}$. Because an RDA is not available for calcium, we used the Adequate Intake (AI) for calcium during pregnancy based on age ( $\leq 18$ years, $1300 \mathrm{mg} ; 19-50$ years, $1000 \mathrm{mg})^{24}$ to assess adequacy of calcium intake. Percentage of energy from fat in the diet, based on recommendations from the Dietary Guidelines for Americans ${ }^{7}$, was our seventh component.

The DQI-P's final component relates to meal/snack patterning. The Institute of Medicine recommends that women should follow a meal pattern of three meals and snacks during gestation ${ }^{25}$. In addition, we have previously reported that meal patterns of pregnant women and the frequency of food intake during pregnancy are relevant to the relationship between maternal nutritional status and pre-term birth ${ }^{26}$

\section{DQI-P scoring}

We based the DQI-P on an 80-point scale, with each of the eight components contributing 10 possible points. We chose to weigh each of the components equally and on an additive scale because the literature lacked information on the relative impact that these dietary constituents have on pregnancy or birth outcomes. To assess adequacy of intake of the first six components, we calculated percentage of the recommendation met as a continuous variable, with a minimum of $0 \%$ to a maximum of $100 \%$. This proportion was multiplied by 10 to obtain a continuous DQI-P score for each of the three food group components, each ranging from 0 to 10 . Percentage of energy as fat and meal pattern were scored in a categorical manner with the optimal fat intake ( $\leq 30 \%$ of calories) and 
meal/snack pattern ( 3 meals and $\geq 2$ snacks per day) receiving a perfect score of 10 (see Table 1 ).

\section{Statistical analysis}

We examined the distribution of each of the DQI-P components within the population and calculated mean values for components by DQI-P score category. Mean values of selected nutrients not included in the index were also calculated by DQI-P score category. We tested for statistically significant trends in DQI-P components and non-DQI-P components across groups of DQI-P score categories. Student's $t$-tests and analysis of variance were used to compare mean index components by sociodemographic characteristics. Stata version 6.0 (StataCorp, College Station, TX) was used for all statistical analysis.

\section{Results}

The mean DQI-P score for this population was 56.0 (standard deviation 12.0) out of 80 possible points. Table 2 shows the mean values of the DQI-P components for the sample and by DQI-P score category. Of the 2063 women in the sample, $11.3 \%$ scored 40 or below, $8.2 \%$ scored between 41 and 45, 11.5\% scored between 46 and 50, 14.4\% scored between 51 and 55, 15.4\% scored between 56 and 60, 16.0\% scored between 60 and 65, 14.3\% scored between 66 and 70, and $8.9 \%$ scored at or above 71 . Table 2 illustrates that the index captures overall variation in quality of the diet. As indicated by the statistically significant increasing trend for all components $(P<$ 0.05 ), diet consistently improved quantitatively as DQI-P score increased. Women in the highest DQI-P score group consumed the most adequate amounts of grains, vegetables and fruits, had the highest intakes of folate, iron and calcium, and consumed the lowest percentage of energy as fat. Women who scored above 70 on the DQI-P had scores reflecting a diet with the most desirable meal pattern. For folate and iron, the percentage of women who consumed less than the Estimated Average Requirement $(\mathrm{EAR})^{21,23}$, and therefore have a probable risk of inadequacy ${ }^{20}$, decreased significantly as DQI-P score increased (data not shown). However, even in the highest DQI-P score category, 16\% of women consumed less than the EAR for iron.

When examining number of servings of grains, fruits and vegetables by energy intake group, the pattern of increasing number of servings with increasing index score was also observed (data not shown). The least variability in servings of grains, fruits and vegetables from lowest to highest DQI-P score was seen in the lowest energy intake group. For grains - even at the highest DQI-P scores women on average did not meet the recommended number of servings.

To further test the internal consistency of this index, we compared relevant food and nutrient values not included as components (protein, dietary fibre, \% of energy from saturated fat and $\%<$ EAR for vitamins $\mathrm{A}, \mathrm{B}_{6}, \mathrm{C}$ and $\mathrm{E}$, zinc, niacin, riboflavin and thiamin) across the range of DQI-P scores (data not shown). For all aforementioned micronutrients, there was a statistically significant decreasing trend in the proportion of women $<$ EAR across index score categories. That is, the $\%$ of individuals with a high probability of having inadequate intakes decreased as DQI-P score increased. For \% of energy from saturated fat, there was a statistically significant downward trend, such that \% of energy from saturated fat decreased as DQI-P score increased. Total grams of protein and dietary fibre increased as index score increased as well. This index was moderately associated with total energy intake. The Pearson correlation coefficient between total energy intake and the DQI-P score was $r=0.48$. Four of the components (grains, folate, iron, calcium) were moderately associated with energy intake ( $r=0.49$ to 0.70$)$.

The DQI-P also detected differences in diet quality by maternal sociodemographic factors (Table 3). Women who were $>350 \%$ of poverty, nulliparous, older and better

Table 2 Mean values of Diet Quality Index for Pregnancy (DQI-P) components by DQI-P score category

\begin{tabular}{|c|c|c|c|c|c|c|c|c|c|}
\hline \multirow[b]{2}{*}{ Variable } & \multirow{2}{*}{$\begin{array}{c}\text { Sample } \\
\text { mean } \pm S D\end{array}$} & \multicolumn{8}{|c|}{ DQI-P score category } \\
\hline & & $\leq 40$ & $41-45$ & $46-50$ & $51-55$ & $56-60$ & $61-65$ & $66-70$ & $>70$ \\
\hline Number of subjects & 2063 & 234 & 171 & 238 & 300 & 320 & 333 & 298 & 184 \\
\hline$\%$ Recommended grain servings per day ${ }^{1 \star}$ & $39.0 \pm 19.0$ & 25.2 & 31.2 & 32.7 & 36.7 & 40.0 & 41.6 & 45.9 & 58.4 \\
\hline$\%$ Recommended vegetable servings per day ${ }^{1 *}$ & $95.4 \pm 68.2$ & 44.3 & 62.2 & 70.7 & 76.9 & 96.5 & 110 & 134 & 163 \\
\hline$\%$ Recommended fruit servings per day ${ }^{1 *}$ & $127 \pm 108$ & 48.2 & 75.9 & 97.3 & 105 & 117 & 153 & 189 & 220 \\
\hline Folate intake as $\% \mathrm{RDA}^{2 \star}$ & $105 \pm 58.2$ & 44.3 & 65.3 & 71.7 & 88.6 & 109 & 118 & 147 & 187 \\
\hline Iron intake as \% RDA* & $74.1 \pm 39.5$ & 34.1 & 49.5 & 53.0 & 63.8 & 77.0 & 83 & 101 & 124 \\
\hline Calcium intake as $\% \mathrm{Al}$ for age ${ }^{*}$ & $125.8 \pm 73.7$ & 61.2 & 86.4 & 93.5 & 115 & 135 & 144 & 168 & 187 \\
\hline$\%$ of energy from total fat ${ }^{\star}$ & $33.5 \pm 6.4$ & 37.5 & 37.2 & 34.8 & 34.0 & 33.7 & 31.8 & 30.8 & 29.1 \\
\hline Meal pattern score ${ }^{3 \star}$ & $7.8 \pm 3.8$ & 5.1 & 6.3 & 6.8 & 7.6 & 7.9 & 8.7 & 9.4 & 9.9 \\
\hline
\end{tabular}

SD - standard deviation; RDA - Recommended Dietary Allowance; Al - Adequate Intake.

* Trend across DQI-P score categories is statistically significant $(P<0.05)$.

${ }^{1}$ Based on Food Guide Pyramid ${ }^{8}$ recommendations for diets containing $\leq 1600,1601-<1900,1900-<2500,2500-<2800$ and $\geq 2800$ kcal

${ }^{2}$ Based on Food Guide Pyramide as dietary folate equivalents.
Folate

${ }^{3}$ Scoring based on the following categories: 3 meals/2 snacks $=10$ points; 3 meals/0-1 snack(s) or 2 meals/2 snacks = 5 points; 2 meals/0-1 snack(s) or $1 \mathrm{meal} / \mathrm{snacks}=0$ points. 


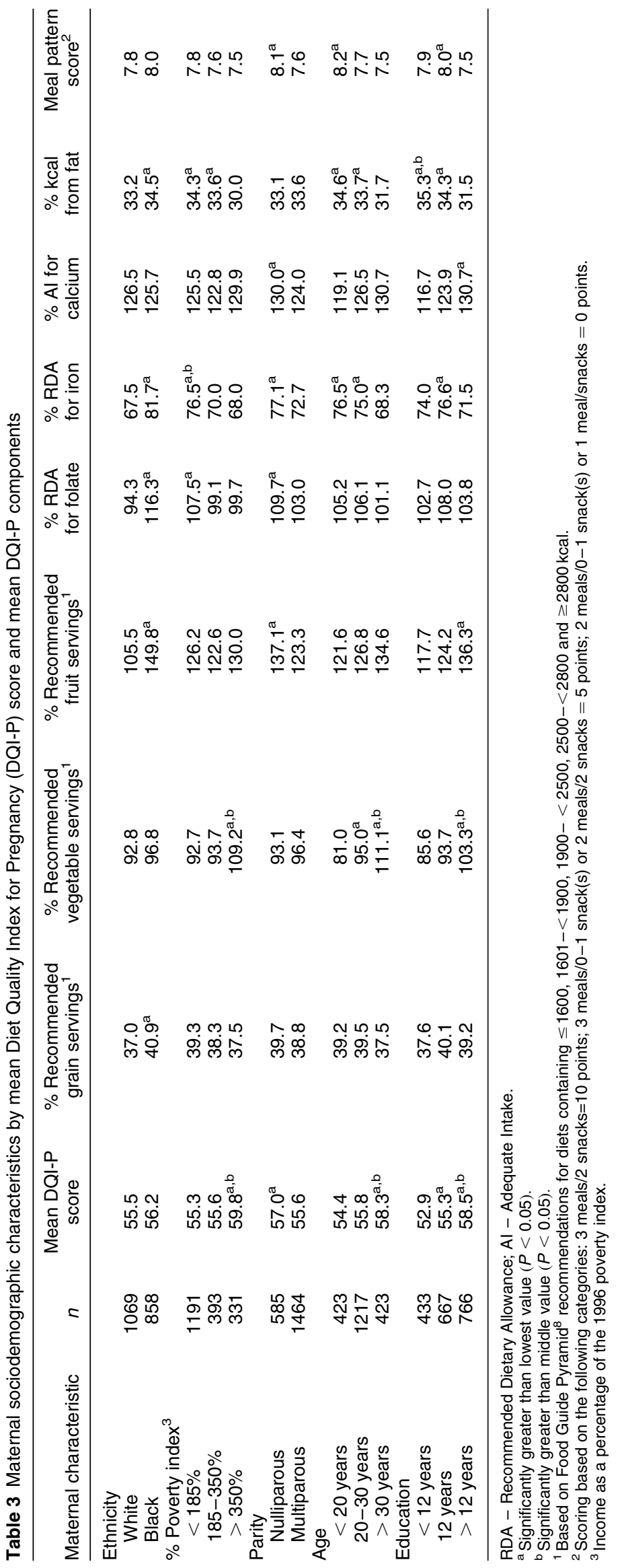


educated had significantly higher overall DQI-P scores. On average, more black women met the recommended number of servings of grains than white women. Higher percentages of recommended vegetable servings were consumed by higher-income, older and better-educated women. Black and nulliparous women as well as women who completed some college education consumed a higher percentage of recommended fruit servings. Regardless of energy intake, better-educated, older and higher-income women consumed one to two additional servings of vegetables compared with their counterparts (data not shown).

Greater percentages of recommended intakes of folate and iron were seen among black, low-income and nulliparous women. Higher iron intakes were also seen among women who graduated high school and were less than 30 years old. Women who were white, $>350 \%$ of poverty, older and better educated more often consumed a diet lower in fat. Women who were nulliparous, young and high school graduates had scores reflecting a more ideal meal/snack pattern. There were no meaningful differences in intake of calcium.

\section{Discussion}

This paper describes an attempt to create a composite measure of diet quality for the pregnant state - a time in which great emphasis is placed on eating a healthy diet. The Diet Quality Index for Pregnancy was developed based on the latest dietary recommendations, and included both food and nutrient components as well as a component based on meal pattern. We based the first three components on food groups for two reasons. First, these food groups provide a recognisable standard upon which people could base the quality of their diets. Second, these food groups captured many important dietary constituents, such as vitamins $\mathrm{A}$ and $\mathrm{C}, \mathrm{B}$ vitamins and dietary fibre, which were not included in this DQI-P directly.

Dietary folate was included as a component because of its importance for the prevention of neural tube defects ${ }^{27}$, promotion of birthweight ${ }^{28}$ and potential relationship with pre-term birth ${ }^{28}$. We included total calcium intake as a component rather than the Pyramid's milk, yoghurt and cheese group because calcium in the diet is often contributed by foods other than dairy, especially among those women who are lactose-intolerant. Similarly, we chose to use iron intake rather than the Food Guide Pyramid's meat, poultry, fish, eggs and beans food group because we have previously shown that the five foods that contribute most to iron intake in this population are grains fortified with iron (i.e. ready-to-eat cereals, white breads) and not animal products ${ }^{29}$. Although poor zinc intake has been shown to be associated with adverse birth outcomes $^{30,31}$, we did not include zinc as a component in the DQI-P because it was highly correlated with iron $(r=0.9)$.
Furthermore, since our results showed that as DQI-P score increased, the percentage of women $<$ EAR for zinc decreased, it is clear that the DQI-P captured zinc intake without including it as a component.

Our inclusion of a dietary fat component was based on the Dietary Guidelines for Americans ${ }^{7}$, which recommend consumption of a diet containing $\leq 30 \%$ of calories from fat. This recommendation is appropriate for pregnant and non-pregnant states. Finally, the Institute of Medicine ${ }^{25}$ gives specific recommendations for the number of eating occasions during pregnancy; thus the meal/snack pattern score was also included in this index.

We did not include as DQI-P components a number of dietary constituents that have been used in other indices of diet quality ${ }^{3-5}$. Dietary variety and dietary moderation scores, while important aspects of the diet, were not included because we felt that, if added, their complexity would limit the use of the DQI-P in public health settings. Furthermore, the Dietary Guidelines for Americans ${ }^{7}$ do not yet quantify 'varied' or 'moderate' dietary intakes. Thus, we felt our own definition would be somewhat arbitrary. Prior indices have included dietary cholesterol and \% of energy from saturated fat. However, our data showed that cholesterol was highly correlated with total fat intake $(r=$ 0.8), an existing component of the DQI-P. Also, our results showed that DQI-P scores reflected saturated fat intakes without including this nutrient specifically as a component. In contrast, our index was consistent with other indices in that we did not include nutrient intake from dietary supplements. The goal of creating the DQI-P was to assess diet in its most traditional sense. If one wishes to measure total nutrient intake, nutrient intake from supplements should then be assessed.

The DQI-P quantitatively differentiated diets, and thus appears to be an appropriate tool for the assessment of diet quality in pregnancy. The high intakes of calcium in this population were a result of frequent consumption of milk ${ }^{29}$, which is not unusual in a sample of pregnant women. Our one concern dealt with the reporting of very low intakes of grain products in this population. Although we included mixed dishes such as pizza, pasta and mixed dishes with meat and/or cheese in the grain food group, the FFQ underestimated grain servings. Recently, one study showed that grains were reported on an FFQ with lower validity than other food groups ${ }^{32}$. It is possible that grain intakes were simply lower than expected, but because the literature lacks data describing how well pregnant women meet Food Guide Pyramid recommendations, we are unable to compare our results with others.

DQI-P score and four of its components were moderately correlated with energy intake. Consequently, some may suggest that the index itself or some of its components should be replaced by total energy intake. Yet, diets high in total energy are not always nutrientdense diets; instead, they may be diets high in fat and simple sugars. Therefore, we feel that the DQI-P 
components are essential in directly assessing adequacy of food and nutrient intakes.

To our knowledge, this paper is the first to describe a composite measure of diet quality for pregnancy that incorporates both foods and nutrients. Carmichael et al. ${ }^{33}$ created a diet quality index based on seven components: intakes of iron, vitamin $\mathrm{B}_{6}$, calcium, vitamin $\mathrm{A}$, folate, percentage of calories from fat and percentage of calories from sweets. For micronutrients women were given a score of 0 if they consumed less than the 10th percentile, 1 if they consumed within the range of the 10th-90th percentile, and 2 if they consumed greater than the 90th percentile for each micronutrient, with opposite scoring for macronutrients. Scores for all components were summed and could range from 0 to 14 . Two other studies have developed measures of diet quality for pregnancy ${ }^{34,35}$, but have also based their indices on adequacy of nutrient intake only. These prior studies have found relationships between diet quality and birth and/or pregnancy outcomes ${ }^{33-35}$. Future research will determine if DQI-P scores are associated with health outcomes as well. Nonetheless, it is important to note the distinctions that must be made in the purpose of these measures. Previous studies examined the effect of nutrient deficiencies (and, in one case ${ }^{33}$, macronutrient excesses) on risk of adverse outcomes, whereas the DQI-P was designed to assess the quality of diet compared with national recommendations for pregnancy.

When comparing the intakes of blacks and whites, Loris et $a .^{36}$ reported similar results for intakes of vegetables and calcium, but contrary to our study, found no ethnic differences in intakes of iron, grains and fruits. Unlike our findings, intakes of iron, calcium and folate have been shown to increase with increasing income $^{37}$ and social class ${ }^{38}$ in British pregnant women. Food group intake in pregnant women has not been studied by income. However, in a nationally representative sample of women of childbearing age, fruit and vegetable intake in low-income women was shown to be significantly lower than in higher-income women ${ }^{39}$. In our pregnant population we only saw this association with vegetables.

While many studies suggest pregnant teenagers consume inadequate servings of important food groups ${ }^{40,41}$ and have inadequate intake of nutrients ${ }^{42}$, few studies have compared intakes of older and younger women. Our findings comparing different age groups of pregnant women have been consistent with those of Endres et $a l .{ }^{41}$ for intakes of folate and vegetables, but inconsistent for intakes of grains, fruits, iron and calcium. Food group intake by education groups has not been reported in pregnant populations. In general, the reported association between maternal education and calcium, iron and folate intakes has been inconsistent ${ }^{36,43,44}$. To our knowledge, differences in dietary intake by parity or differences in percentage of calories from fat or meal pattern by any sociodemographic groupings have not been reported in the literature.

None of the papers described above, including ours, describes a nationally representative sample of pregnant women; thus, discrepancies in results could represent cultural or regional differences in dietary intake. Furthermore, most of the aforementioned investigations used small sample sizes, while the strength of our study is our large, diverse cohort.

We believe that this Diet Quality Index for Pregnancy is reproducible for use in other research studies. All of the components of this index are relatively easy to quantify, especially as a new calculation for dietary folate equivalents is now available ${ }^{45}$. We based our calculation of DFEs on food weight because this method is similar to a model used previously ${ }^{46}$. Although the most accurate method employed by the USDA is to separate foods into commodities, we did not have this capability and felt that using the proportion of weight contributed by fortified foods was the next best option. For situations in which DFEs cannot be calculated in this or another manner, we feel that, because of its importance during pregnancy, total dietary folate intake without accounting for increased bioavailability of folic acid as a percentage of the RDA should remain a component in the DQI-P. As computerised dietary assessment tools are now being used more often in public health sites such as Special Supplemental Nutrition Program for Women, Infants, and Children (WIC) clinics, indices such as the DQI-P can be applicable in non-research settings.

Although the DQI-P is based on a food-frequency questionnaire, it should be applicable for use with other dietary assessment tools that capture usual dietary intake, such as multiple 24-hour recalls or food records. Additionally, we believe that although this index was created based on dietary intake in the second trimester, the components can be applied to dietary intake in any trimester of pregnancy because recommendations do not differ.

For all people, including pregnant women, it is essential that we examine all aspects of the diet rather than limiting our assessment of nutritional status on the basis of one indicator, which does not explain the complexity of the diet. Because this index is based on national recommendations, the DQI-P may be a useful research and public health tool for evaluating the overall diet quality of pregnant women.

\section{References}

1 Institute of Medicine, National Academy of Sciences. Nutrition during Pregnancy: Weight Gain and Nutrient Supplements. Washington, DC: National Academy Press, 1990.

2 Koblinsky MA. Beyond maternal mortality - magnitude, interrelationship, and consequences of women's health, pregnancy-related complications and nutritional status on 
pregnancy outcomes. Int. J. Gynaecol. Obstet. 1995; 48: S21-32.

3 Haines PS, Siega-Riz AM, Popkin BM. The Diet Quality Index Revised: a measurement instrument for populations. J. Am. Diet. Assoc. 1999; 99: 697-704.

4 Patterson RE, Haines PS, Popkin BM. Diet Quality Index: capturing a multidimensional behavior. J. Am. Diet. Assoc. 1994; 94: 57-64.

5 Kennedy ET, Ohis F, Carlson S, Fleming K. The Healthy Eating Index: design and applications. J. Am. Diet. Assoc. 1995; 95: 1103-8.

6 Basiotis PP, Guthrie JF, Bowman SA, Welsh SO. Construction and evaluation of a Diet Status Index. Farm. Econ. Nutr. Rev. 1995; 8(2): 2-13.

7 US Department of Agriculture, Department of Health and Human Services. Dietary Guidelines for Americans, 5th ed [Online]. Available at http://www.usda.gov/cnpp/DietGd. pdf. Accessed 29 January 2001.

8 Shaw A, Fulton L, Savis C, Hogbin M. Using the Food Guide Pyramid: A Resource for Nutrition Educators [Online] Available at http://www.nal.usda.gov/fnic/Fpyr/guide.pdf. Accessed 29 January 2001.

9 Food Surveys Research Group, Agriculture Research Service (ARS). 1994 Continuing Survey of Food Intakes by Individuals (CSFII) 1994 [CD-ROM]. Disks 1 and 2. Riverdale, MD: ARS, 1994.

10 US Department of Agriculture. USDA Nutrient Data Base for Standard Reference, Release \#12. Riverdale, MD: Agriculture Research Service, 1998

11 Block G, DiSogra C. WIC Dietary Assessment Validation Study. Final Report. Alexandria, VA: US Department of Agriculture, Food and Nutrition Service, 1995.

12 Block G, Coyle LM, Hartman AM, Scoppa SM. Revision of dietary analysis software for the health habits and history questionnaire. Am. J. Epidemiol. 1994; 139: 1190-6.

13 Block G, Thompson FE, Hartman AM, Larkin FA, Guire KE. Comparison of two dietary questionnaires validated against multiple dietary records collected during a 1-year period. J. Am. Diet. Assoc. 1992; 92: 686-93.

14 Block G, Woods M, Potosky A, Clifford C. Validation of a selfadministered diet history questionnaire using multiple diet records. J. Clin. Epidemiol. 1990; 43: 1327-35.

15 Subar AF, Thompson FE, Smith AF, Jobe JB, Ziegler RG. Improving food frequency questionnaires: a qualitative approach using cognitive interviewing. J. Am. Diet. Assoc. 1995; 95: 781-8.

16 National Cancer Institute. Dietsys Software Version 4.0 for Block-NCI HHHQ [Online]. Available at http://www. nutritionquest.com.

17 National Cancer Institute (NCI). 5-A-Day for Better Health. RFA No. CA-92-17. Bethesda, MD: NCI, 1992.

18 National Research Council, Subcommittee on the 10th Edition of the RDAs, Food and Nutrition Board, Commission on Life Sciences. Recommended Dietary Allowances, 10th ed. Washington, DC: National Academy Press, 1989.

19 Kopp-Hoolihan LE, van Loan MD, Wong WW, King JC. Longitudinal assessment of energy balance in well-nourished, pregnant women. Am. J. Clin. Nutr. 1999; 69 697-704.

20 Institute of Medicine, Food and Nutrition Board. Dietary Reference Intakes: Applications in Dietary Assessment. Washington, DC: National Academy Press, 2000.

21 Institute of Medicine, Food and Nutrition Board. Dietary Reference Intakes for Thiamin, Riboflavin, Niacin, Vitamin B-6, Folate, Vitamin B-12, Pantothenic Acid, Biotin, and Choline. Washington, DC: National Academy Press, 1998.

22 Yates AA, Schlicker SA, Suitor CW. Dietary Reference Intakes: the new basis for recommendations for calcium and related nutrients, B vitamins, and choline. J. Am. Diet. Assoc. 1998; 98: 699-706.
23 Institute of Medicine, Food and Nutrition Board. Dietary Reference Intakes for Vitamin A, Vitamin K, Arsenic, Boron, Chromium, Copper, Iodine, Iron, Manganese, Molybdenum, Nickel, Silicon, Vanadium, and Zinc. Washington, DC: National Academy Press, 2001.

24 Institute of Medicine, Food and Nutrition Board. Dietary Reference Intakes for Calcium, Phosphorus, Magnesium, Vitamin D, and Fluoride. Washington, DC: National Academy Press, 1997.

25 Institute of Medicine, Committee on Nutritional Status during Pregnancy and Lactation, Food and Nutrition Board Subcommittee for Clinical Application Guide. Nutrition during Pregnancy and Lactation: An Implementation Guide. Washington, DC: National Academy Press, 1992.

26 Siega-Riz AM, Herrmann TS, Savitz DA, Thorp J. The frequency of eating during pregnancy and its effect on preterm delivery. Am. J. Epidemiol. 2001; 153: 647-52.

27 Lawrence KM, James N, Miller MH, Tennant GB, Campbell H. Double-blind randomized controlled trial of folate treatment before conception to prevent recurrence of neural-tube defects. $\mathrm{Br}$. Med. J. 1981; 282: 1509-11.

28 Scholl TO, Hediger ML, Schall JI, Khoo C, Fisher RL. Dietary and serum folate: their influence on the outcome of pregnancy. Am.J. Clin. Nutr. 1996; 63: 520-5.

29 Siega-Riz AM, Bodnar LM, Savitz DA. What are pregnant women eating? Nutrient and food group differences by race. Am. J. Obstet. Gynecol. 2002; 186: 480-6.

30 Goldenberg RL, Tamura T, Neggers Y, Copper RL, Johnston $\mathrm{KE}, \mathrm{DuBard} \mathrm{MB}$, et al. The effect of zinc supplementation on pregnancy outcome. J. Am. Med. Assoc. 1995; 274: 463-8.

31 Neggers YH, Cutter GR, Acton RT, Alvarez JO, Bonner JL, Goldenberg RL, et al. A positive association between maternal serum zinc concentration and birth weight. $A m$. J. Clin. Nutr. 1990; 51: 678-84.

32 Rosner B, Gore R. Measurement error correction in nutritional epidemiology based on individual foods, with application to the relation of diet to breast cancer. $\mathrm{Am}$. J. Epidemiol. 2001; 154: 827-35.

33 Carmichael SL, Shaw GM, Selvin S, Schaffer DM. Diet quality and risk of neural tube defects [abstract]. Am. J. Epidemiol. 2000; 151(11): S6.

34 Philipps C, Johnson NE. The impact of quality of diet and other factors on birth weight of infants. Am. J. Clin. Nutr. 1977; 30: 215-25.

35 Burke BS, Beal VA, Kirkwood SB, Stuart HC. Nutrition studies during pregnancy. Am.J. Obstet. Gynecol. 1943; 46 $38-52$.

36 Loris P, Dewey KG, Poirier-Brode K. Weight gain and dietary intake of pregnant teenagers. J. Am. Diet. Assoc. 1985; 85(10): 1296-305.

37 Haste FM, Brooke OG, Anderson HR, Bland JM, Peacock JL. Social determinants of nutrient intake in smokers and nonsmokers during pregnancy. J. Epidemiol. Community Health 1990; 44: 205-9.

38 Haste FM, Brooke OG, Anderson HR, Bland JM, Shaw JM, Griffin J, Peacock JL. Nutrient intakes during pregnancy: observations on the influence of smoking and social class. Am. J. Clin. Nutr. 1990; 51: 29-36.

39 Block G, Abrams G. Vitamin and mineral status of women of childbearing potential. Ann. N.Y. Acad. Sci. 1993; 678: $244-54$.

40 Skinner JD, Carruth BR, Pope J, Varner L, Goldberg D. Food and nutrient intake of white, pregnant adolescents. J. Am. Diet. Assoc. 1992; 92(9): 1127-9.

41 Endres J, Dunning S, Poon S, Welch P, Duncan H. Older pregnant women and adolescents: nutrition data after enrollment in WIC. J. Am. Diet. Assoc. 1987; 87(8): 1011-9.

42 Lenders CM, McElrath TF, Scholl TO. Nutrition in adolescent pregnancy. Curr. Opin. Pediatr. 2000; 12: 291-6. 
43 Suitor CW, Gardner JD, Feldstein ML. Characteristics of diet among culturally diverse group of low-income pregnant women. J. Am. Diet. Assoc. 1990; 90: 543-9.

44 Rush D, Sloan NL, Leighton J, Alvir JM, Horvitz DG, Seaver WBV, et al. Longitudinal study of pregnant women. Am. J. Clin. Nutr. 1988; 48: 439-83.
45 NDS-R Software, (C) Regents of the University of Minnesota Nutrition Coordinating Center. All Rights Reserved.

46 Lewis CJ, Crane NT, Wilson DB, Yetley EA. Estimated folate intakes: data updated to reflect food fortification, increased bioavailability, and dietary supplement use. Am. J. Clin. Nutr. 1999; 70: 189-207. 